\title{
Metal-Phenolic Supramolecular Gelation
}

Authors: Md. Arifur Rahim, ${ }^{1}$ Mattias Björnmalm, ${ }^{1}$ Tomoya Suma, ${ }^{1}$ Matthew Faria, ${ }^{1}$ Yi Ju, ${ }^{1}$ Kristian Kempe, ${ }^{1, \dagger}$ Markus Müllner, ${ }^{1, \ddagger}$ Hirotaka Ejima, ${ }^{1, \S}$ Anthony D. Stickland, ${ }^{2}$ and Frank Caruso $^{1 *}$

\section{Affiliations:}

${ }^{1}$ ARC Centre of Excellence in Convergent Bio-Nano Science and Technology, and the Department of Chemical and Biomolecular Engineering, The University of Melbourne, Parkville, Victoria 3010, Australia

${ }^{2}$ Particulate Fluids Processing Centre, Department of Chemical and Biomolecular Engineering, The University of Melbourne, Parkville, Victoria 3010, Australia

*Correspondence to: fcaruso@unimelb.edu.au

†Present address: ARC Centre of Excellence in Convergent Bio-Nano Science and Technology, Monash Institute of Pharmaceutical Sciences, Monash University, Parkville, Victoria 3052, Australia

$\ddagger$ Present address: School of Chemistry, The University of Sydney, Sydney, New South Wales 2006, Australia

§Present address: Institute of Industrial Science, The University of Tokyo, 4-6-1 Komaba, Meguro-ku, Tokyo, Japan

Abstract: Materials assembled via coordination interactions between naturally abundant polyphenols and metals are of interest for a wide range of applications including crystallization, catalysis, and drug delivery. Such an interest has led to the development of thin films with tunable, dynamic properties; however, creating bulk materials remains a challenge. Here, we report a class of metallogels formed by direct gelation between inexpensive, naturally abundant tannic acid and group(IV) metal ions. The metallogels exhibit diverse properties, including selfhealing and transparency, and can be doped with various materials via in situ co-gelation. The robustness and flexibility, combined with the ease, low cost, and scalability of the coordinationdriven assembly process make these metallogels potential candidates for chemical, biomedical, and environmental applications. 
Functional materials in nature are assembled under ambient conditions from a limited selection of components; however, they display remarkably complex and dynamic behavior. ${ }^{[1-3]} \mathrm{A}$ prominent example is mussel byssal threads, which form hard but flexible coatings through metal-phenolic coordination. ${ }^{[4]}$ We recently demonstrated that metal-phenolic complexation between different metals and phenolic ligands can be used to assemble conformal coatings, yielding thin films and particles with tailored properties. ${ }^{[5-7]}$ Metal-phenolic complexation is also of interest for the assembly of bulk materials, such as supramolecular biomaterials and gels: ${ }^{[8-10]}$ 3,4-dihydroxyphenylalanine-modified peptides and polyethylene glycol were complexed with dior trivalent transition metals (e.g., $\mathrm{Fe}^{\mathrm{III}}$ ) to assemble metal-phenolic gels. ${ }^{[11-15]}$ However, the assembly of such gels requires the use of synthetic, pre-synthesized macromolecular building blocks. ${ }^{[11-15]}$ While polyphenol-based gels can be assembled using non-coordination interactions, for example between tannic acid (TA) and polymers, ${ }^{[16,17]}$ direct gelation between naturally occurring, unmodified polyphenols and metal ions is yet to be demonstrated.

Herein, we report the simple and rapid assembly of metal-phenolic gels (or metallogels) via the direct gelation of naturally occurring and inexpensive TA and group(IV) metal ions. The chemistry underlying this gelation is specific to group(IV) transition metals. We use the gelation between TA and titanium(IV) $\left(\mathrm{Ti}^{\mathrm{iV}}\right)$ as a primary example to demonstrate the formation of metallogels and their properties. These metallogels can form in different organic solvents and aqueous solutions through simple mixing at a range of molar ratios. Furthermore, these gels can be engineered to exhibit tunable mechanical properties, optical transparency, injectability, moldability, shape persistence, adhesiveness, and self-healing properties, and to have stability in the $\mathrm{pH}$ range of $2-10$. Both TA and titanium are known to be highly biocompatible, with TA 
being a natural antioxidant and titanium being extensively used in clinical implants. ${ }^{[18]}$ This gelation process is robust and the metallogels can be doped in situ via co-gelation with various materials to endow application-specific properties.

The polyphenolic structure of TA consists of five digalloyl ester groups covalently attached to a central glucose core (Figure 1a). The galloyl functional groups of TA provide multitopic chelating sites that can coordinate a range of transition metals. ${ }^{[6]}$ Solutions of TA and $\mathrm{Ti}^{\mathrm{IV}}$ formed gels after mixing, and such gelation occurred in diverse organic and aqueous solvents including $N$-methyl-2-pyrrolidone (NMP), $N, N$-dimethylformamide (DMF), and water (Figure 1b-h). The organogels are denoted as "TA-Ti ${ }^{\mathrm{IV}}$-OG (solvent)" and the hydrogels as "TA$\mathrm{Ti}^{\mathrm{IV}}$-HG”.

The orange-red $\mathrm{TA}-\mathrm{Ti}^{\mathrm{IV}}$ sol (Figure 1d) formed an optically transparent dark red gel upon standing at room temperature $\left(21-24{ }^{\circ} \mathrm{C}\right)$, as demonstrated by the tube/vial inversion test (Figure 1e-h). The gelation time could be tuned from minutes to weeks by changing the concentration of $\mathrm{TA}$ and the molar ratio of $\mathrm{TA}$ to $\mathrm{Ti}^{\mathrm{IV}}$ (Figures $\mathrm{S} 1$ and S2 in the Supporting Information). The gelation behavior could be also controlled using sonication and heating-both processes reduced the gelation time (Figure S1). Notably, no gelation was observed for the TA$\mathrm{Ti}^{\mathrm{IV}}$-OG (NMP) system prepared at a $\mathrm{TA} / \mathrm{Ti}^{\mathrm{IV}}$ molar ratio of $1: 1$, even when the $\mathrm{TA}$ concentration was increased to $20 \mathrm{wt}$ \% (Figure S1). Furthermore, the combination of TA with other first row transition metals (under similar conditions) did not result in gelation (Figures S3 and S4), suggesting that the direct gelation is specific to group(IV) metal ions. In particular, the gelation of $\mathrm{TA}$ and $\mathrm{Fe}^{\mathrm{III}}$ was unsuccessful (Figure S3), which is consistent with a previous study. ${ }^{[16]}$ However, $\mathrm{Fe}^{\mathrm{III}}$ and other metals could be incorporated in situ in the TA-Ti ${ }^{\mathrm{IV}}$ matrix via co-gelation (Figure 1g). This allowed the fabrication of a wide range of functional materials, 
some of which are explored below. The gelation behavior of $\mathrm{TA}-\mathrm{Ti}^{\mathrm{IV}}$-HG prepared at different TA concentrations and stoichiometries is presented in Figure S2. The gelation times of the TA$\mathrm{Ti}^{\mathrm{IV}}$-HG system were faster than those of the $\mathrm{TA}-\mathrm{Ti}^{\mathrm{IV}}-\mathrm{OG}$ system (Figures $\mathrm{S} 1$ and $\mathrm{S} 2$ ). For example, using a $\mathrm{TA} / \mathrm{Ti}^{\mathrm{IV}}$ molar ratio of $1: 4$ and $5 \mathrm{wt} . \% \mathrm{TA}$, the gelation times were $\sim 1$ min and $1 \mathrm{~h}$ for $\mathrm{TA}-\mathrm{Ti}^{\mathrm{IV}}$-HG and $\mathrm{TA}-\mathrm{Ti}^{\mathrm{IV}}-\mathrm{OG}$ (NMP), respectively (Figures $\mathrm{S} 1$ and $\mathrm{S} 2$ ).

UV-Visible absorption spectroscopy performed on the $\mathrm{TA} / \mathrm{Ti}^{\mathrm{IV}}$ sols showed the characteristic ligand-to-metal charge transfer (LMCT) band of $\mathrm{TA}-\mathrm{Ti}^{\mathrm{IV}}$ coordination (bis/tristype in terms of the galloyl chelating sites) at $375 \mathrm{~nm}$ (Figure 1j). ${ }^{[19]}$ Conversely, TA and $\mathrm{Ti}^{\mathrm{IV}}$ solutions were featureless in the same spectral region $(350-550 \mathrm{~nm})$. The sol-to-gel transition of the $\mathrm{TA}-\mathrm{Ti}^{\mathrm{IV}}-\mathrm{OG}$ (NMP) system was probed in situ by dynamic oscillatory rheology experiments (Figure 1k). Initially, liquid-like (viscous) properties were observed, as evident from the higher loss modulus $\left(\mathrm{G}^{\prime \prime}\right)$ relative to the storage modulus $\left(\mathrm{G}^{\prime}\right)$ (Figure $\left.1 \mathrm{k}\right)$. As time increased, the phase shift $(\delta)$ continued to decrease and $\mathrm{G}^{\prime}$ increased, indicating the solid-like (elastic) properties of the system. The sol-gel transition occurred when $\mathrm{G}^{\prime}>\mathrm{G}^{\prime \prime}$ after the $\mathrm{G}^{\prime} / \mathrm{G}^{\prime \prime}$ cross-over point at $\sim 20$ min. When accounting for the time required for the experimental setup, this corresponds well with the observed gelation time ( $\sim 0 \mathrm{~min}$ ) determined from the tube/vial inversion test.

To further investigate the $\mathrm{TA}-\mathrm{Ti}^{\mathrm{IV}}$-OG (NMP) gel structure, X-ray photoelectron spectroscopy (XPS) was performed on the dried gels (Figure 2a,b). C 1s, O 1s, and Ti 2p peaks were detected in the survey spectra (Figure 2a). From the Ti 2p core-level photoelectron spectrum (Figure $2 \mathrm{~b}$ ), $\mathrm{Ti} 2 \mathrm{p}_{3 / 2}$ and $\mathrm{Ti} 2 \mathrm{p}_{1 / 2}$ peaks were observed at binding energies of 459.3 and $465 \mathrm{eV}$, respectively, and were assigned to $\mathrm{Ti}^{\mathrm{IV}}$ species in the gel. ${ }^{[20]}$ Additionally, these results indicate the absence of redox reactions between $\mathrm{TA}$ and $\mathrm{Ti}^{\mathrm{IV}}$ during gelation. As expected from 
the asymmetric multitopic structure of TA, the dried gels were amorphous, as determined by Xray diffraction (XRD) (Figure 2c).

The $\mathrm{TA}-\mathrm{Ti}^{\mathrm{IV}}$ gel system exhibits a combination of notable properties including shape persistence, high adhesive strength, load-bearing capability, and self-healing ability. These properties could be tuned by adjusting the composition and processing parameters of the gels. For example, the mechanical properties of the gels were changed by modifying the stoichiometry and concentration for a given solvent (Figures $2 \mathrm{~d}$ and S5). At a TA/Ti ${ }^{\mathrm{IV}}$ molar ratio of 1:3, $\mathrm{G}^{\prime}$ of $\mathrm{TA}^{\mathrm{Ti}}{ }^{\mathrm{IV}}$-OG (NMP) increased from $\sim 300$ to $\sim 5000 \mathrm{~Pa}$ with increasing concentrations, as determined from the time sweep tests (conducted at a frequency of $1 \mathrm{~Hz}$ and $1 \%$ strain). At each concentration studied, $G^{\prime}$ was greater than $G^{\prime \prime}$, thus suggesting the true gel nature of the materials prepared. The gels are moldable and demonstrate shape persistence behavior (Figure 2e-h). Shape-persistent gels with a variety of shapes, including cylindrical, spherical, toroidal, triangular, and tubular, were obtained through molding, and no apparent changes in the shape or size were observed over time (up to 3 months). The gels demonstrated high load-bearing capabilities and could hold loads of $\sim 70$ times greater than their own weight (i.e., a $0.45 \mathrm{~g}$ metallogel could hold a 30 g load) (Figure 2i). Additionally, the adhesive capability of the gel was demonstrated by forming a thin $(1 \mathrm{~mm})$ gel layer between two glass plates-the gel prevented the glass plates from slipping (Figure $2 \mathrm{j}, \mathrm{k}$ ). In contrast to covalently bonded chemical gels, the dynamic nature of coordination bonding can endow self-healing properties in the resulting material. ${ }^{[11,16]}$ Thus, the self-healing properties of the $\mathrm{TA}^{-\mathrm{Ti}^{\mathrm{IV}}}$ gel system were investigated using a fracture-recovery test (Figure 2l-o). Gels formed in a glass Petri dish were fractured by a sharp blade and then allowed to stand unperturbed. The fractures healed over time-any visible traces of the fractures disappeared completely within $2 \mathrm{~h}$. 
The TA- $\mathrm{Ti}^{\mathrm{IV}}$ gels are non-cytotoxic (Figure S6) and are compatible with a range of environments and post-assembly processing treatments (Figure 3). For example, the gels could be made injectable (Figure 3a). Manual force on the piston of a syringe induced extrusion of the gel without clogging. Because of the high strain, the extruded gel was initially deformed but quickly self-repaired (Figure 3a,b). Furthermore, the gels could be dried to create monolithic xerogels and aerogels (Figure 3c,d). Moderate gel shrinkage was observed for the aerogels, whereas a substantial decrease in volume was observed for the xerogels. However, in both cases, volume shrinkage was homogenous without damage to the overall structure. The stability of the $\mathrm{TA}^{-\mathrm{Ti}^{\mathrm{IV}}}$-HG at various $\mathrm{pH}$ values was also investigated (Figure $3 \mathrm{~m}$ ). The hydrogel system was stable in the $\mathrm{pH}$ range of $2-10$, suggesting strong coordination bonding between $\mathrm{TA}$ and $\mathrm{Ti}^{\mathrm{IV}}$ in the gel. However, the hydrogel disassembled when subjected to pHs greater than 10 (Figure S7).

A notable feature of $\mathrm{TA}-\mathrm{Ti}^{\mathrm{IV}}$ gel systems is their ability to incorporate diverse functional materials in the gel matrix via in situ co-gelation. The materials to be embedded can be mixed with the gel components in the initial $\mathrm{TA}-\mathrm{Ti}^{\mathrm{IV}}$ sol, and co-gelation generates composite gel materials: we investigated $\mathrm{Fe}^{\mathrm{III}}$, vanadium(III) $\left(\mathrm{V}^{\mathrm{III}}\right)$, iron oxide magnetic nanoparticles $\left(\mathrm{Fe}_{3} \mathrm{O}_{4}\right.$ MNP), cobalt ferrite magnetic nanoparticles (Co/Fe MNP), graphene oxide (GO), poly(3,4ethylenedioxythiophene)-poly(styrenesulfonate) (PEDOT:PSS), and multiwalled carbon nanotubes (MWCNT) (Figures 1g and 3e-j). Gels loaded with Co/Fe MNP exhibited magnetism and those loaded with GO were conductive (Figure 3k,l). In contrast, the pristine gels did not exhibit any magnetic response or conductivity behavior.

The robust nature of the prepared gel systems and the ability to add diverse dopants led us to consider more complex additives. The close relationship between the coordination chemistry used to form the $\mathrm{TA}-\mathrm{Ti}^{\mathrm{IV}}$ gels and metal-organic frameworks (MOFs) prompted us to 
investigate if MOF crystallization could be realized in situ during formation of the $\mathrm{TA}-\mathrm{Ti}^{\mathrm{IV}}$ gels. As a proof of concept, MOF-5 and HKUST- 1 crystallization ${ }^{[21]}$ within the TA-Ti ${ }^{\mathrm{IV}}$ gel system was examined-crystallization and gelation occurred simultaneously without inhibiting each other (Figure 3n-q). As a control, MOF structures in bulk were prepared for comparison (Figure S8). Differences in the morphologies of the MOF particles (e.g., MOF-5 needles of lengths of $\sim 40-60 \mu \mathrm{m}$ in the gel compared with MOF-5 needles of lengths of $100-150 \mu \mathrm{m}$ in solution) could be attributed to the spatial influence of the gel matrix on MOF crystallization. The MOFgel composites are structures that contain both crystalline (MOF) and amorphous (metallogel) coordination networks. Co-formation of such multilevel hybrid structures is rare and provides both new insight into this type of chemistry and the potential for new applications.

In general, the gelation mechanism underlying the formation of metallogels is not completely understood. ${ }^{[22-25]}$ However, we hypothesize that the high oxidation state and formal charge of $\mathrm{Ti}^{\mathrm{IV}}$ play a significant role in the solvent-trapping process of the gelation, ${ }^{[24-27]}$ in addition to coordinative crosslinking, enabling the formation of gel networks. This may explain why $\mathrm{Fe}^{\mathrm{III}}$ does not form a gel in the presence of TA despite exhibiting the strongest known coordination interactions with catechol-type ligands. ${ }^{[19,28]}$ Additionally, this explanation predicts that other group(IV) metals would form gels upon combination with TA, as indicated by the positive results obtained from preliminary experiments involving $\mathrm{Zr}^{\mathrm{IV}}$ (Figure S9). The current results thus provide new insight into the formation of dynamic, coordination-driven metallogels, and potentially represent a versatile platform for the development of new materials for diverse applications. We are currently exploring such gels for their potential application in drug crystallization and delivery, catalysis, and metal sequestration and environmental remediation. 


\section{Methods}

Methods and associated references are available in the Supporting Information.

Keywords: organic-inorganic hybrid composites • chelates • polyphenols • titanium

Acknowledgements: This research was supported by the Australian Research Council (ARC) under the Australian Laureate Fellowship (FL120100030) scheme, and the ARC Centre of Excellence in Convergent Bio-Nano Science and Technology (Project CE140100036). This work was performed in part at the Materials Characterisation and Fabrication Platform at the University of Melbourne and the Victorian Node of the Australian National Fabrication Facility.

\section{Competing financial interests}

The authors declare no competing financial interest.

\section{References}

[1] C. Ortiz, M. C. Boyce, Science 2008, 319, 1053-1054.

[2] J. Aizenberg, P. Fratzl, Adv. Mater. 2009, 21, 187-188.

[3] K. Ariga, J. Li, J. Fei, Q. Ji, J. P. Hill, Adv. Mater. 2016, 28, 1251-1286.

[4] M. J. Harrington, A. Masic, N. Holten-Andersen, J. H. Waite, P. Fratzl, Science 2010, 328, 216-220.

[5] H. Ejima, J. J. Richardson, K. Liang, J. P. Best, M. P. van Koeverden, G. K. Such, J. Cui, F. Caruso, Science 2013, 341, 154-157.

[6] J. Guo, Y. Ping, H. Ejima, K. Alt, M. Meissner, J. J. Richardson, Y. Yan, K. Peter, D. von Elverfeldt, C. E. Hagemeyer, F. Caruso, Angew. Chem. Int. Ed. 2014, 53, 5546-5551; Angew. Chem. 2014, 126, 5652-5657.

[7] M. A. Rahim, K. Kempe, M. Müllner, H. Ejima, Y. Ju, M. P. van Koeverden, T. Suma, J. A. Braunger, M. G. Leeming, B. F. Abrahams, F. Caruso, Chem. Mater. 2015, 27, 58255832.

[8] M. J. Webber, E. A. Appel, E. W. Meijer, R. Langer, Nat. Mater. 2015, 15, 13-26.

[9] M.-O. M. Piepenbrock, G. O. Lloyd, N. Clarke, J. W. Steed, Chem. Rev. 2010, 110, 19602004.

[10] X. Du, J. Zhou, J. Shi, B. Xu, Chem. Rev. 2015, 115, 13165-13307.

[11] N. Holten-Andersen, M. J. Harrington, H. Birkedal, B. P. Lee, P. B. Messersmith, K. Y. C. Lee, J. H. Waite, Proc. Natl. Acad. Sci. U. S. A. 2011, 108, 2651-2655.

[12] N. Holten-Andersen, A. Jaishankar, M. J. Harrington, D. E. Fullenkamp, G. DiMarco, L. He, G. H. McKinley, P. B. Messersmith, K. Y. C. Lee, J. Mater. Chem. B 2014, 2, 2467- 
2472.

[13] Q. Li, D. G. Barrett, P. B. Messersmith, N. Holten-Andersen, ACS Nano 2016, 10, 13171324.

[14] H. Ceylan, M. Urel, T. S. Erkal, A. B. Tekinay, A. Dana, M. O. Guler, Adv. Funct. Mater. 2013, 23, 2081-2090.

[15] S. C. Grindy, R. Learsch, D. Mozhdehi, J. Cheng, D. G. Barrett, Z. Guan, P. B. Messersmith, N. Holten-Andersen, Nat. Mater. 2015, 14, 1210-1216.

[16] M. Krogsgaard, A. Andersen, H. Birkedal, Chem. Commun. 2014, 50, 13278-13281.

[17] M. Shin, J. H. Ryu, J. P. Park, K. Kim, J. W. Yang, H. Lee, Adv. Funct. Mater. 2015, 25, 1270-1278.

[18] P. Tengvall, I. Lundström, Clin. Mater. 1992, 9, 115-134.

[19] M. J. Sever, J. J. Wilker, Dalton Trans. 2004, 1061-1072.

[20] R. Inde, M. Liu, D. Atarashi, E. Sakai, M. Miyauchi, J. Mater. Chem. A 2016, 4, 17841791.

[21] N. Stock, S. Biswas, Chem. Rev. 2012, 112, 933-969.

[22] M. Cametti, M. Cetina, Z. Džolić, Dalton Trans. 2015, 44, 7223-7229.

[23] A. Y.-Y. Tam, V. W.-W. Yam, Chem. Soc. Rev. 2013, 42, 1540.

[24] L. A. Estroff, A. D. Hamilton, Chem. Rev. 2004, 104, 1201-1218.

[25] T. D. Hamilton, D.-K. Bučar, J. Baltrusaitis, D. R. Flanagan, Y. Li, S. Ghorai, A. V. Tivanski, L. R. MacGillivray, J. Am. Chem. Soc. 2011, 133, 3365-3371.

[26] H. Liang, Z. Zhang, Q. Yuan, J. Liu, Chem. Commun. 2015, 51, 15196-15199.

[27] B. Xing, M.-F. Choi, B. Xu, Chem. Eur. J. 2002, 8, 5028-5032.

[28] Z. Xu, Sci. Rep. 2013, 3, 2914. 

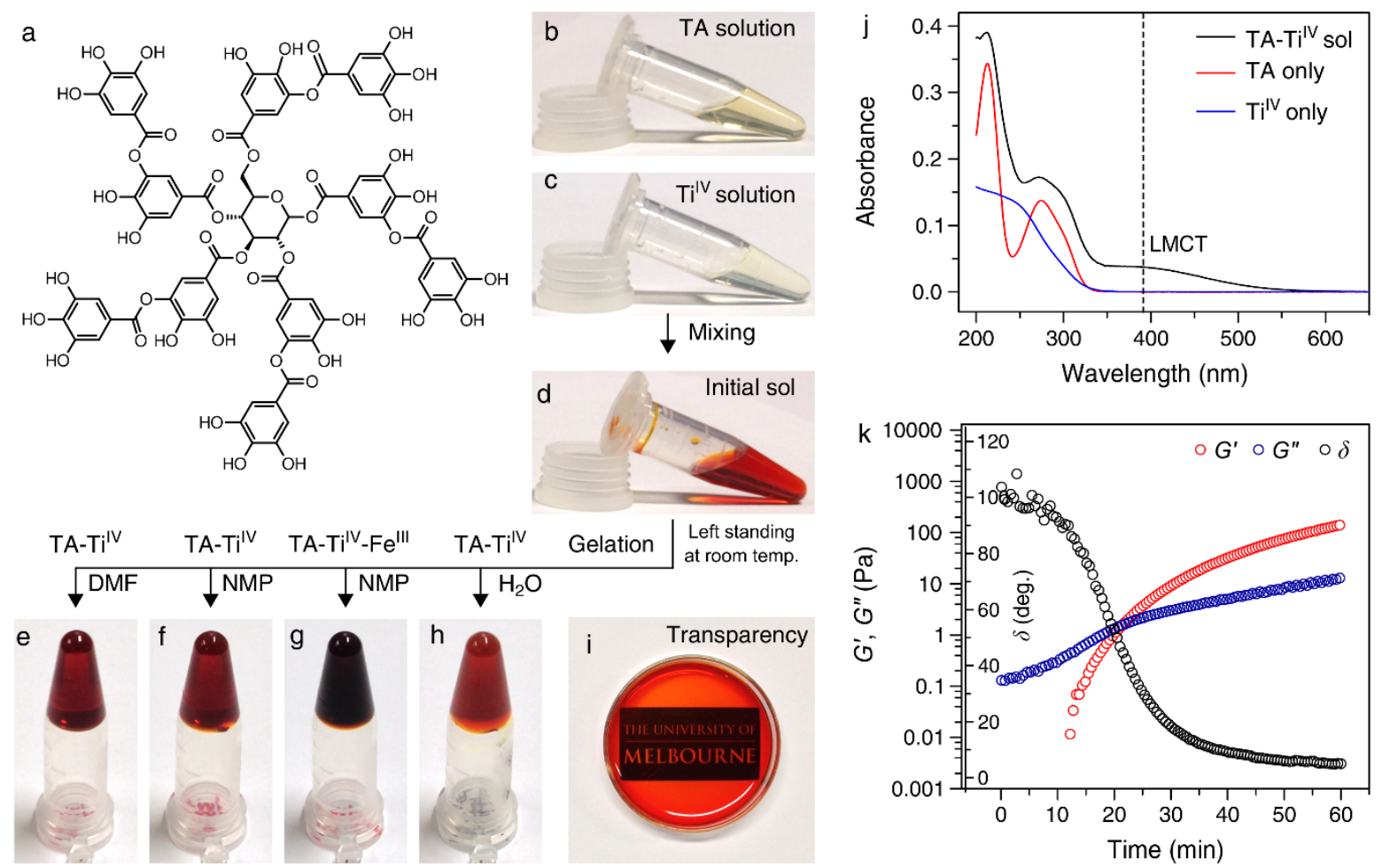

Figure 1. Gelation of the $\mathrm{TA}-\mathrm{Ti}^{\mathrm{IV}}$ gel system. a) Structure of $\mathrm{TA}$ containing multiple chelating sites (galloyl groups). Mixing of the (b) $\mathrm{TA}$ solution with (c) $\mathrm{Ti}^{\mathrm{IV}}$ solution produces (d) an orange-red $\mathrm{TA}-\mathrm{Ti}^{\mathrm{IV}}$ sol. e-h) Subsequent gelation in different solvents. i) $\mathrm{TA}-\mathrm{Ti}^{\mathrm{IV}}-\mathrm{OG}$ (NMP) thin gel prepared on a glass Petri dish. j) UV-Visible absorption spectrum of a TA-Ti ${ }^{\mathrm{IV}}$ sol. k) Dynamic oscillatory rheological measurement showing the sol-gel transition of the TA-Ti ${ }^{\mathrm{IV}}$ gel system. 

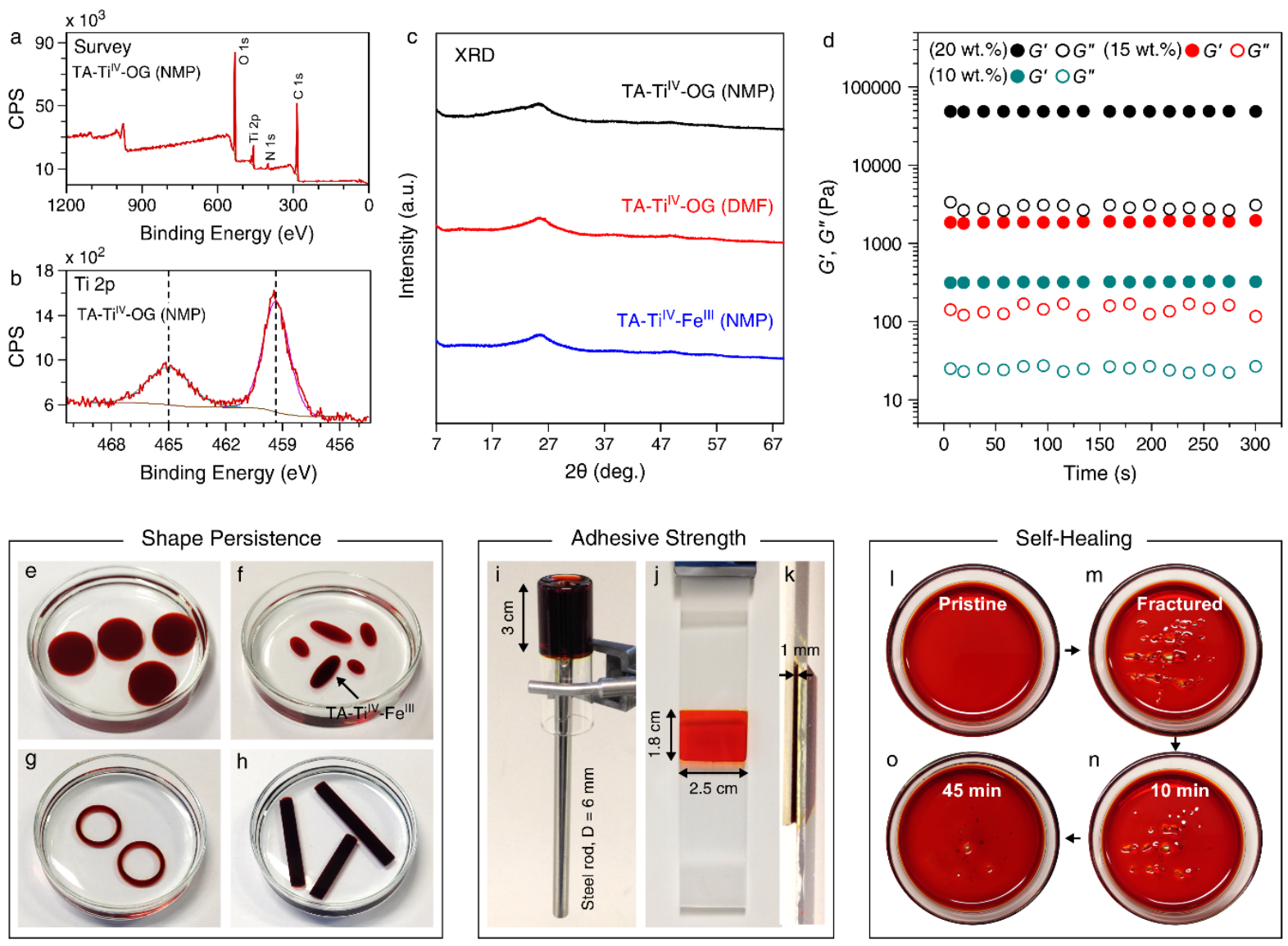

Figure 2. Characterization and properties of the TA-Ti ${ }^{\mathrm{IV}}$ gels. a,b) XPS spectra of the dried TA$\mathrm{Ti}^{\mathrm{IV}}$-OG (NMP) gels. c) XRD patterns of the dried TA-Ti ${ }^{\mathrm{IV}}$ and $\mathrm{TA}-\mathrm{Ti}^{\mathrm{IV}}-\mathrm{Fe}^{\mathrm{III}}$ gels. d) Dynamic oscillatory rheological measurements of $\mathrm{TA}-\mathrm{Ti}^{\mathrm{IV}}-\mathrm{OG}$ (NMP) prepared at different $\mathrm{TA}$ concentrations $\left(\mathrm{TA} / \mathrm{Ti}^{\mathrm{IV}}=1: 3\right)$. e-h) Shape of the gels: (e) disc-shaped, (f) oval, (g) toroidal, and (h) tubular. i) The load-bearing capability of the gels is demonstrated by holding an inverted steel rod in place. j,k) The high adhesive strength of the gels is demonstrated by preventing two glass plates from slipping. $1-0)$ Self-healing properties of the gel $\left(\mathrm{TA}-\mathrm{Ti}^{\mathrm{IV}}(\mathrm{NMP}), \mathrm{TA} / \mathrm{Ti}^{\mathrm{IV}}=1: 2\right)$ monitored over time. 

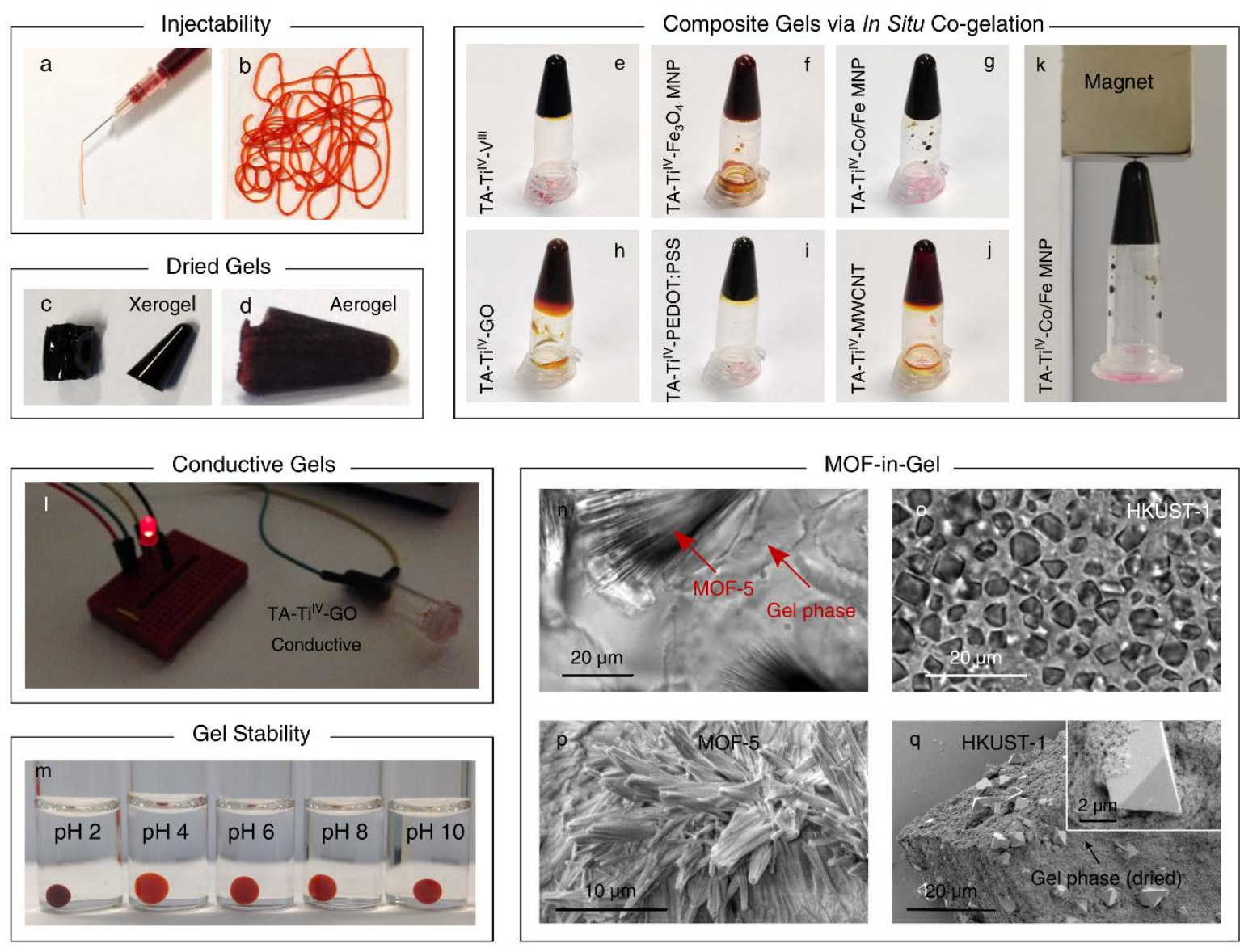

Figure 3. Properties of the TA-Ti ${ }^{\mathrm{IV}}$ gel system. a,b) Injectable gel. c,d) Monolithic xerogels and aerogels prepared by (c) solvent evaporation and (d) supercritical drying of the wet gels, respectively. e-j) Composite gels prepared by incorporating diverse materials via in situ cogelation without affecting the fundamental gelation process. k) Magnetic behavior of the TA$\mathrm{Ti}^{\mathrm{IV}}$-Co/Fe MNP system. l) Conductivity property of the $\mathrm{TA}-\mathrm{Ti}^{\mathrm{IV}}-\mathrm{GO}$ system, which is capable of powering a light-emitting diode. m) Stability tests performed on the $\mathrm{TA}-\mathrm{Ti}^{\mathrm{IV}}$-HG system that was exposed to different $\mathrm{pH}$ values. n,o) Differential interference contrast (DIC) images of MOF-5 and HKUST-1 crystallization in the $\mathrm{TA}^{\mathrm{Ti}} \mathrm{Ti}^{\mathrm{IV}}$ gel system. p,q) Scanning electron microscopy (SEM) characterization of MOF-5 and HKUST-1 crystallization in the TA-Ti ${ }^{\mathrm{IV}}$ gel system. 


\section{Table of Contents Graphic}

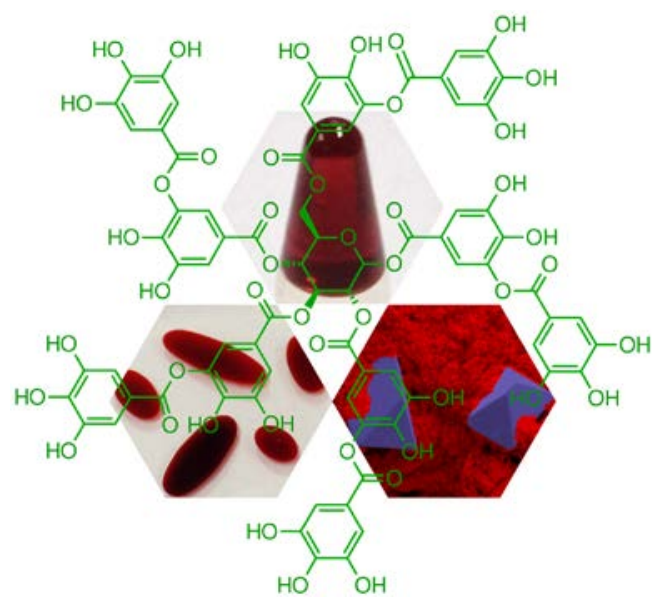

Ti-Tannic Gels: The direct gelation of the natural polyphenol tannic acid via coordination-driven supramolecular assembly with titanium(IV) is reported. These metallogels exhibit a range of properties, including transparency, injectability, moldability, self-healing, shape persistence, adhesiveness, and tunable mechanics. 


\section{University Library}

\section{- M M N E R VA A gateway to Melbourne's research publications}

Minerva Access is the Institutional Repository of The University of Melbourne

Author/s:

Rahim, MA;Bjornmalm, M;Suma, T;Faria, M;Ju, Y;Kempe, K;Muellner, M;Ejima, H;Stickland, $A D$;Caruso, $F$

Title:

Metal-Phenolic Supramolecular Gelation

Date:

2016-10-24

Citation:

Rahim, M. A., Bjornmalm, M., Suma, T., Faria, M., Ju, Y., Kempe, K., Muellner, M., Ejima, H., Stickland, A. D. \& Caruso, F. (2016). Metal-Phenolic Supramolecular Gelation. ANGEWANDTE CHEMIE-INTERNATIONAL EDITION, 55 (44), pp.13803-13807. https:// doi.org/10.1002/anie.201608413.

Persistent Link:

http://hdl.handle.net/11343/120181 